\title{
RIESGOS CLIMÁTICOS EN LA CIUDAD DE ZARAGOZA
}

\author{
Fernando LÓPEZ MARTÍN \\ Departamento de Geografía y Ordenación del Territorio \\ Universidad de Zaragoza
}

\begin{abstract}
Resumen: Los riesgos de origen climático se producen en la ciudad de Zaragoza provocando numerosos siniestros con repercusiones personales, materiales y económicas. Para su estudio hemos utilizado los datos de intervenciones del servicio de Bomberos y los datos diarios del INM, con ellos se ha intentado fijar unos umbrales de los riesgos de origen climático( viento y precipitación) en Zaragoza.
\end{abstract}

Palabras Clave: Ciudad, intervenciones, precipitación, riesgo climático, umbrales, viento, Zaragoza.

\begin{abstract}
The risk of climatic origin happen in the city of Zaragoza causing lot of dammage with personal, material and economic repercussions. To implement the study, both the interventions of the Firemen Service and INM daily data have been taken into account. Out of them it has been intended to fixe the threshold of climatic origin risk (wind and precipitation) in Zaragoza.
\end{abstract}

Key words: City, interventions, precipitation, risk, threshold, wind, Zaragoza.

\section{INTRODUCCIÓN}

Con frecuencia cuando se hace referencia a los riesgos climáticos, pensamos en los grandes episodios de carácter catastrófico que se producen en el espacio geográfico, casi siempre inducidos por la inadecuada intervención humana que, por ejemplo, han llevado a cuantiosas pérdidas económicas como las producidas entre 1990-1993 que han ascendido a 154.594 millones de dólares. Pero a pesar de ello rara vez centramos nuestra atención sobre un ámbito como el de la ciudad, en la que por ser totalmente artificial, creemos que no puede ser afectada por los riesgos climáticos, lo cual evidentemente es un error.

No podemos olvidar que cuando hablamos del espacio urbano, nos estamos refiriendo al lugar donde habitan más de 4.000 millones de personas, por lo que los 
riesgos climáticos que se producen en este entorno afectan a más de la mitad de la población del mundo.

Si bien es cierto que la mayoría de las ciudades del Tercer Mundo carecen de una red de infraestructuras (red de alcantarillado, conducciones, bomberos, etc) que puedan mitigar las situaciones de riesgo de origen climático, no es menos cierto que en las ciudades de nuestro entorno, cuando se producen situaciones excepcionales, por su cantidad o por su intensidad, se desbordan todas las infraestructuras pasivas y activas para la prevención, ocasionando situaciones de riesgo personal, material y económico. Sobre todo respecto a este último apartado, es cada vez más frecuente la utilización de seguros en edificios, viviendas e incluso los Ayuntamientos los utilizan, frente a daños causados por riesgos de origen climático, teniendo que recurrir a las certificaciones del INM para fijar las primas y las compensaciones correspondientes.

Por ello, podemos afirmar, que la ciudad es un medio que por su carácter artificial, realiza una función protectora frente a los riesgos climáticos; red de abastecimiento frente a escasas precipitaciones, niebla y nieve atenuada por la "isla de calor", exceso de precipitaciones corregidas por la red de alcantarillado, etc. Pero precisamente por el carácter de espacio muy ocupado (personas, construcciones, etc) cuando la catástrofe se produce, las repercusiones económicas son mayores que otros espacios menos humanizados.

Es claro, que se deben establecer las medidas de prevención (red de alcantarillado, protección civil, bomberos, etc) en las ciudades para intentar minimizar los efectos de estas situaciones de riesgo. Entre estas medidas, se encuentra la de un conocimiento de los efectos de los riesgos de origen climático en la ciudad, para intentar prevenir y tomar las oportunas medidas correctoras. Hemos creído, que un punto de partida de este conocimiento en la ciudad de Zaragoza, podría ser el estudio del viento y de las precipitaciones, fenómenos meteorológicos causantes de la mayor parte de los riesgos en nuestra ciudad, e intentar fijar unos umbrales de peligrosidad de cada uno de estos meteoros en el caso concreto de Zaragoza.

\section{FUENTES Y MÉTODO}

La información utilizada como fuente más fiable para el análisis del riesgo climático en la ciudad de Zaragoza, ha sido la relativa a las intervenciones del Cuerpo de Bomberos de la ciudad. Los datos han podido ser obtenidos gracias a la colaboración de D. Augusto García Hegardt, Jefe del Servicio Contra Incendio, Salvamento y Protección Civil, que gentilmente facilitó la información. 
Los datos de intervenciones se encuentran clasificados, según el criterio de Protección Civil, en las memorias del año 1995 y primer semestre de 1996, en 102 claves, de las que se seleccionaron, dentro del capítulo de salvamentos, aquellas relacionadas directamente con los factores meteorológicos, quedando restringidas a 6 , agrupándose de la siguiente forma:

- Actuaciones por salvamento: agua (agua, achique, contención y desvío).

- Actuaciones por salvamento: riesgos naturales (lluvias, riadas, avenidas, huracanes, vendavales, nieve, heladas).

- Actuaciones por salvamento: ruinas y hundimiento (saneado de construcciones, consolidación, caída de árboles, carteles, farolas y otros).

En total se han analizado 8.832 siniestros, correspondientes al año 1995 y primer semestre de 1996, de los que 636 se deben a las claves señaladas de salvamento, pero como este análisis sólo, podría enmascarar intervenciones que no se debieran a causas climáticas, se recurrió al repaso del resumen diario de intervenciones, analizando el contenido de cada una de ellas, hasta deslindar las provocadas directa o indirectamente por un origen climático, de las que no lo son, en total aparecen 352 intervenciones.

En cuanto a las fuentes meteorológicas necesarias para establecer las relaciones oportunas, como indicadores de riesgo, se han utilizado los partes SYNOP correspondientes al aeropuerto de Zaragoza, analizándose 14 pulsaciones correspondientes a un total de 29 días, comprobándose que la actuación individual o conjunta del viento y la precipitación, son los responsables de los siniestros, con unos límites más o menos definidos con los que poder establecer la consideración de riesgo climático.

Para un análisis más detallado de éstos meteoros, acudimos al I.N.M. para el estudio de las pulsaciones más significativas a través del dato diario de las estaciones del Aeropuerto y del Observatorio (Parque Primo de Rivera), puesto que el primero era muy válido para el estudio del viento, y el segundo para la precipitación por ser un fenómeno más localizado. De igual forma se decidió utilizar los datos diarios de racha máxima de viento y de intensidad máxima de precipitación como los más significativos para el estudio del riesgo climático en la ciudad. Es muy de destacar la amable colaboración de D.José Manuel Molina Vico, Jefe de Climatología del INM, del Centro Territorial de Aragón, Rioja y Navarra. 
A partir de estos datos henos efectuado un análisis temporal de los siniestros y de las variables climáticas en meses con pulsaciones de siniestros significativas, como son mayo de 1995 y mayo de 1996.

\section{ANÁLISIS TEMPORAL}

Del análisis del Gráfico 1, se puede deducir claramente, una menor incidencia de siniestros, en los meses de invierno y verano, por tanto se establece en ese período $\mathrm{m}$ menor riesgo climático, sobre todo debido a situaciones del Nordeste y Anticiclónicas en invierno, y Anticiclónicas en verano, con un tiempo en general estable y de escasas o nulas precipitaciones. Aumentan los siniestros en primavera, debido a una mayor inestabilidad atmosférica provocada por la entrada de aire húmedo, en las situaciones del Este y de bajas presiones en el Mediterráneo, que puede traer lluvias abundantes de hasta $40 \mathrm{lts} / \mathrm{m}^{2}$ en un día. Este ritmo no se rompe, quizás por el período estudiado, salvo por las fuertes tormentas de convección que pueden aparecer en los meses de verano provocando graves situaciones de riesgo climático.

La ciudad de Zaragoza mantiene una media de $300 \mathrm{~mm}$. de precipitación anual, es decir unas precipitaciones muy escasas y que por su cantidad no serán motivo de riesgo aunque si por su intensidad puntual. Por tanto, lo que es evidente es que el mayor número de siniestros son los provocados por el viento (saneado de construcciones, consolidación, caída de árboles, farolas y otros) que alcanzan al $80 \%$, a pesar de ser una ciudad relativamente preparada para el viento, pues es azotada frecuentemente por el viento del WNW (cierzo), llegando a alcanzar intensidades de $100-120 \mathrm{Km} / \mathrm{hr}$ e incluso rachas de $160 \mathrm{Km} / \mathrm{hr}$ (julio de 1954), que provocan la aparición de siniestros, que mantienen el ritmo marcado de aparición en los meses de primavera y otoño, de igual forma que los provocados por las precipitaciones (achique, contención y desvío). Es evidente que la irregularidad de los fenómenos meteorológicos en la ciudad, aparecerían si se alarga el período estudiado, no debiendo olvidar que también se modifican las condiciones de la ciudad, mejoras en la red de alcantarillado y modernización del caserío, pueden minimizar un riesgo climático que en el pasado creaba gran cantidad de siniestros.

En cuanto a la frecuencia diaria, las intervenciones por las causas señaladas, se sitúan en torno a dos de media, sobre todo por las labores cotidianas de mantenimiento de la ciudad, pero cuando éstas suben a diez (han llegado a 80), suele coincidir con aumentos notables del viento o de las precipitaciones, que con frecuencia forman pulsaciones de dos días, dependiendo de la intensidad del fenómeno climático. Siendo el segundo día para saneamiento de los daños provocados en el primero. 
Gráfico 1

Frecuencia mensual de los siniestros en Zaragoza

(1995-1을

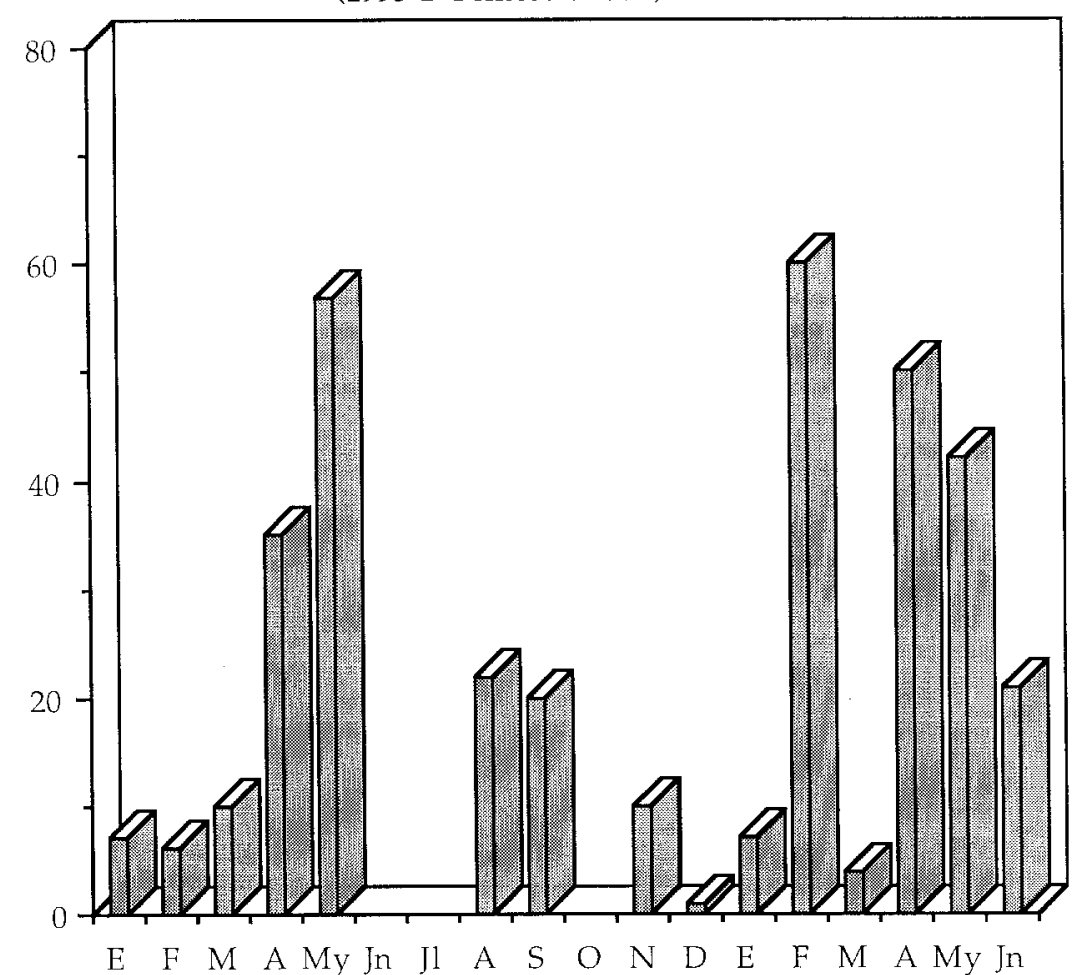

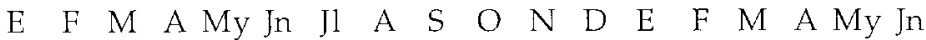

Fuente: Parque Bomberos

Elaboracion Tropia

圆 $\mathrm{N}^{\prime \prime}$ siniestros

Son estos umbrales los que hemos seleccionado como indicadores del riesgo climático en nuestra ciudad, entendiendo como un período excepcional durante el cual "se rompe la marcha habitual y cotidiana de la sociedad" (PITA, 1995)

\section{ESTUDIO DE CASOS}

Como hemos señalado anteriormente, con el fin de establecer las relaciones entre las variables climáticas y las intervenciones del Cuerpo de Bomberos, como indicadores de riesgo, se han estudiado 14 pulsaciones en las que se aprecia un aumento 
notable de la intensidad del viento y las precipitaciones, cruzando estos datos con los siniestros producidos.

En general se observa una diferenciación entre los efectos del viento y de la precipitación, no advirtiéndose diferencias cuando ambos meteoros actúan conjuntamente, a diferencia de lo que se señala a este respecto en el estudio de la ciudad de Madrid (ALMENDROS y ot., 1995).

De la observación del conjunto de datos, se podría señalar que el umbral de riesgo en la ciudad de Zaragoza cuando actúa el viento, se situaría en torno a los $55-60 \mathrm{Km} / \mathrm{hr}$ de intensidad de racha máxima, situándose por encima del señalado para Madrid, de $44 \mathrm{Km} / \mathrm{hr}$.

Respecto a la precipitación, se observa que con intensidades de $25 \mathrm{~mm} / \mathrm{hr}$, el número de siniestros supera la media con amplitud, por supuesto dependiendo del espacio de tiempo que dure la precipitación, aumentando el riesgo si se concentra en unos pocos minutos. Igualmente depende el efecto, en gran medida, de la época del año en que se produce, pues en primavera y otoño intensidades de $20-25 \mathrm{~mm} / \mathrm{hr}$ no provocan demasiadas intervenciones, al encontrarse limpia la red de alcantarillado por 1 a "frecuencia de lluvias", pero sin embargo estas mismas cantidades e incluso menores, producidas con carácter tormentoso en época estival pueden provocar numerosos siniestros, por la obstrucción del alcantarillado, etc.

Para ilustrar la relación de las variables climáticas y las intervenciones, hemos elegido dos meses, mayo de 1995 y mayo de 1996, representativos cada uno de ellos de la relación directa entre viento y precipitación, y los siniestros provocados.

En el mes de mayo de 1996, se produce el día 12 una intensidad de $82 \mathrm{Km} / \mathrm{hr}$ de racha máxima de viento, lo que provocó 21 intervenciones directas, creando una pulsación que se mantuvo el día 13 con 16 intervenciones, a pesar de la disminución de la intensidad del viento. Se puede ver que el día 20 se produjeron rachas de $62 \mathrm{Km} / \mathrm{hr}$ lo que debería haber provocado siniestros, y no fue así, fundamentalmente porque en 1 a pulsación anterior todos aquellos elementos de la ciudad susceptibles de ser afectados por el viento, ya han sido derribados y retirados, lo que parece indicar que también los elementos urbanísticos sufren una acumulación de resistencia, hasta que ceden por el efecto reiterado de rachas intensas, como se puede observar los días 30 y 31 , con intensidades de $62-63 \mathrm{Km} / \mathrm{hr}$, que vuelven a provocar más de 10 intervenciones. 


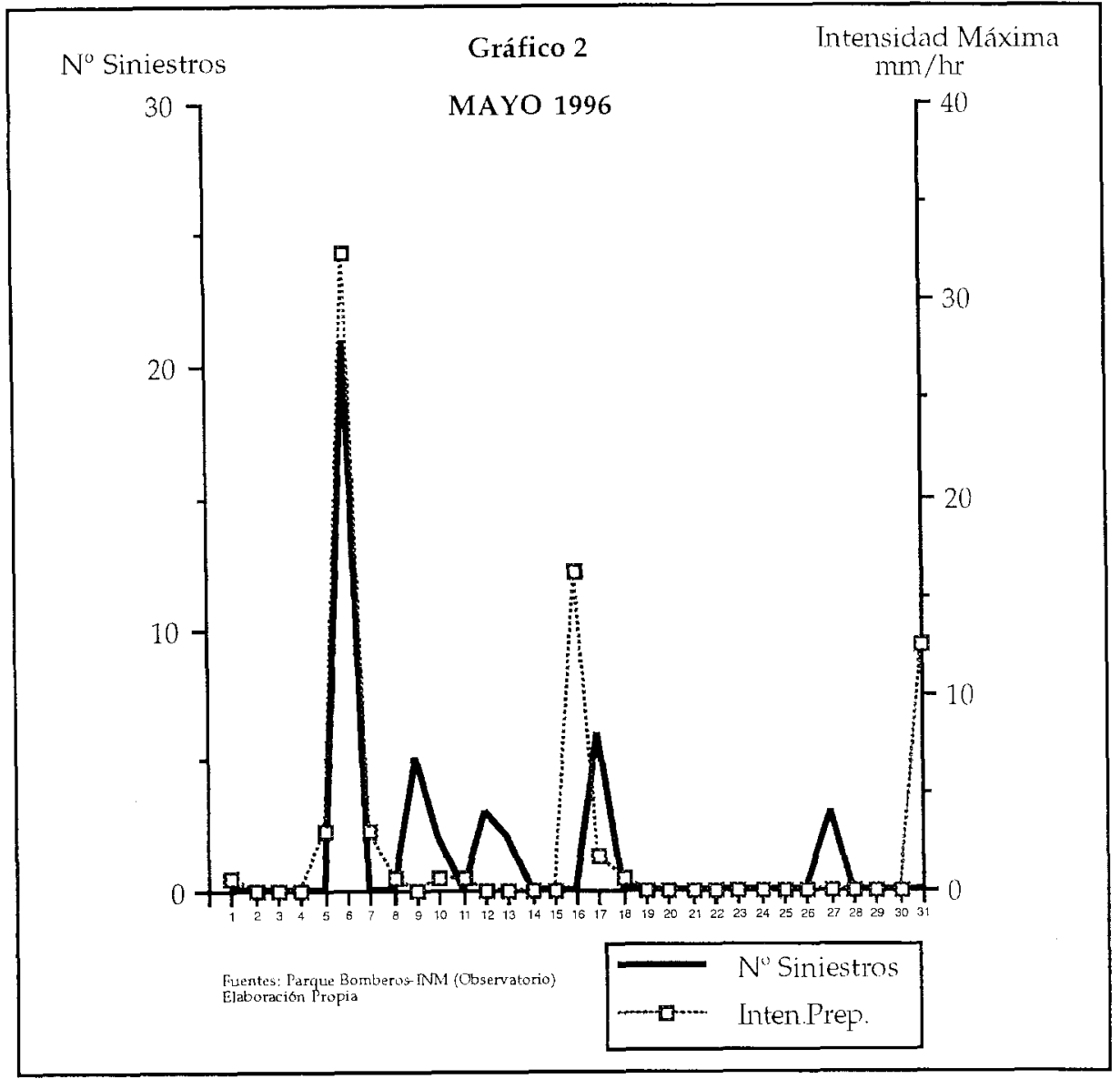

El segundo ejemplo, Gráfico 2, corresponde al mes de mayo de 1996, en el que se observa los efectos provocados por un frente frío asociado a un centro de bajas presiones que estaba situado en las costas de Portugal, situación frecuente en primavera y otoño, provocando fuertes aguaceros. El día 6 se produjo una intensidad máxima de 32,4 $\mathrm{mm} / \mathrm{hr}$, llegando a caer $5,4 \mathrm{~mm} / \mathrm{m}^{2}$ en tan sólo diez minutos, entre las 16,20 hrs y las $16,30 \mathrm{hrs}$, provocando 27 salidas registradas para dar respuestas a las más de 120 llamadas que recibieron los Bomberos. Como se puede observar la pulsación se mantuvo varios días, a pesar de no producirse más precipitaciones, se debió al restablecimiento de la normalidad, sobre todo en la zona del barrio de San José donde la situación se vio agravada por las obras de prolongación de la Avda. Tenor Fleta, creando una verdadera situación de riesgo y de alarma social. 
El día 16 se produjeron intensidades de $16,2 \mathrm{~mm} / \mathrm{hr}$, que en principio no debían provocar siniestros, pero el efecto de acumulación de la tromba del día 6, con el debilitamiento producido en los elementos urbanos, hicieron que se produjeran 8 intervenciones y no así el día 31 con intensidades mayores de $12,1 \mathrm{~mm} / \mathrm{hr}$, cuando ya se habían limpiado redes de alcantarillado y protegido las zonas susceptibles de inundación.

Los fenómenos descritos se distribuyen especialmente, en la ciudad, de tal forma que los barrios más densamente poblados, céntricos y antiguos en su caserío, son los que ofrecen el mayor número de puntos de riesgo (acceso avda. Madrid por túnel, acceso a urgencias H. Miguel Servet, Po Constitución, sótanos zona centro, etc), sin duda los barrios periféricos, con un caserío más moderno y menos denso, suelen verse afectados con mucha menor frecuencia.

\section{CONCLUSIONES}

Parece claro que las fuentes utilizadas, como son las intervenciones del Parque de Bomberos y los datos diarios del INM, aportan una información susceptible de relacionarse para el establecimiento del riesgo climático en la ciudad de Zaragoza.

A pesar de las irregularidades de los fenómenos meteorológicos, podríamos determinar que los umbrales de riesgo climático en la ciudad de Zaragoza, son de 55-60 $\mathrm{Km} / \mathrm{hr}$ de intensidad de racha máxima de viento y de $25 \mathrm{~mm} / \mathrm{hr}$ de intensidad en las precipitaciones.

La fijación de estos umbrales no se presenta sólo como un hecho de investigación, sino que es de aplicación en campos como los seguros (fijación correcta de primas y compensaciones), planificación urbana y planes de Protección Civil, como el Plan de Emergencia Temporal de la ciudad de Zaragoza.

Es evidente que la ciudad de Zaragoza esta más preparada para el viento que para las precipitaciones y que se afecta mucho más la zona centro, con caserío más envejecido. 


\section{BIBLIOGRAFÍA}

ALMENDROS, M.A. y FERNÁNDEZ, F. (1995): "Riesgos de origen climático en la ciudad: el caso de Madrid", En Situaciones de riesgo climático en España, 163-170. Asociación de Geografos Españoles. Jaca (Huesca).

CUADRAT, J.M., DE LA RIVA, J., LÓPEZ MARTÍN, F. y MARTÍ, A. (1993): "El medio ambiente urbano de Zaragoza. Observaciones sobre la "isla de calor". Anales de Geografía de la Universidad Complntense,13. Madrid.

LÓPEZ MARTíN, F. (1995): "Nota sobre la percepción del clima urbano. El ejemplo de la ciudad de Zaragoza". Geographicalia 32, 123-137. Dpto. Geografía y Ordenación del Territorio de la Universidad de Zaragoza. Zaragoza.

ORTEGA ALBA, F. (1991): "Incertidumbre y riesgo natural". Sociedad y Territorio, 99-108. Asoc.Geógrafos Españoles. Valencia.

PITA, F. y AGUILAR (1995): Riesgos naturales. Curso sobre impactos y riesgos climáticos. CEDEX. Madrid. 